\title{
Background studies for acoustic neutrino detection at the South Pole
}

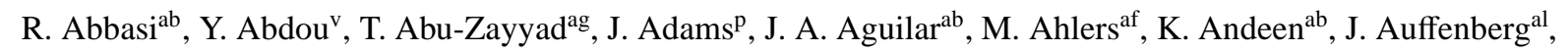
X. Bai ${ }^{\text {ae }}$, M. Baker ${ }^{\mathrm{ab}}$, S. W. Barwick ${ }^{\mathrm{x}}$, R. Bay ${ }^{\mathrm{g}}$, J. L. Bazo Alba ${ }^{\mathrm{am}}$, K. Beattie ${ }^{\mathrm{h}}$, J. J. Beatty ${ }^{\mathrm{r}, \mathrm{s}}$, S. Bechet $^{\mathrm{m}}$,

J. K. Becker ${ }^{\mathrm{j}}$, K.-H. Becker ${ }^{\mathrm{al}}$, M. L. Benabderrahmane ${ }^{\mathrm{am}}$, S. BenZvi $^{\mathrm{ab}}$, J. Berdermann ${ }^{\mathrm{am}, *}$, P. Berghaus ${ }^{\mathrm{ab}}$, D. Berley ${ }^{\mathrm{q}}$,

E. Bernardini ${ }^{\text {am }}$, D. Bertrand ${ }^{\mathrm{m}}$, D. Z. Besson ${ }^{\mathrm{z}}$, D. Bindig ${ }^{\mathrm{al}}$, M. Bissok ${ }^{\mathrm{a}}$, E. Blaufuss ${ }^{\mathrm{q}}$, J. Blumenthal ${ }^{\mathrm{a}}$, D. J. Boersma ${ }^{\mathrm{a}}$,

C. Bohm ${ }^{\text {ah }}$, D. Bose ${ }^{\mathrm{n}}$, S. Böser ${ }^{\mathrm{k}}$, O. Botner ${ }^{\mathrm{ak}}$, J. Braun $^{\mathrm{ab}}$, A. M. Brown ${ }^{\mathrm{p}}$, S. Buitink ${ }^{\mathrm{h}}$, M. Carson ${ }^{\mathrm{v}}$, D. Chirkin ${ }^{\mathrm{ab}}$,

B. Christy ${ }^{\mathrm{q}}$, J. Clem ${ }^{\mathrm{a} e}$, F. Clevermann ${ }^{\mathrm{t}}$, S. Cohen ${ }^{\mathrm{y}}$, C. Colnard ${ }^{\mathrm{w}}$, D. F. Cowen ${ }^{\text {aj,ai }}$, M. V. D' Agostino ${ }^{\mathrm{g}}$,

M. Danninger ${ }^{\text {ah }}$, J. Daughhetee ${ }^{\mathrm{e}}$, J. C. Davis ${ }^{\mathrm{r}}$, C. De Clercq ${ }^{\mathrm{n}}$, L. Demirörs ${ }^{\mathrm{y}}$, T. Denger ${ }^{\mathrm{k}}$, O. Depaepe ${ }^{\mathrm{n}}$, F. Descamps $^{\mathrm{v}}$, P. Desiati ${ }^{\mathrm{ab}}$, G. de Vries-Uiterweerd ${ }^{\mathrm{v}}$, T. DeYoung ${ }^{\mathrm{aj}}$, J. C. Díaz-Vélez ${ }^{\mathrm{ab}}$, M. Dierckxsens ${ }^{\mathrm{m}}$, J. Dreyer ${ }^{\mathrm{j}}$, J. P. Dumm $^{\mathrm{ab}}$,

R. Ehrlich ${ }^{\mathrm{q}}$, J. Eisch ${ }^{\mathrm{ab}}$, R. W. Ellsworth ${ }^{\mathrm{q}}$, O. Engdegård ${ }^{\mathrm{ak}}$, S. Euler ${ }^{\mathrm{a}}$, P. A. Evenson ${ }^{\mathrm{ae}}$, O. Fadiran ${ }^{\mathrm{d}}$, A. R. Fazely ${ }^{\mathrm{f}}$,

A. Fedynitch $^{\mathrm{j}}$, T. Feusels ${ }^{\mathrm{v}}$, K. Filimonov ${ }^{\mathrm{g}}$, C. Finley ${ }^{\text {ah }}$, T. Fischer-Wasels ${ }^{\mathrm{al}}$, M. M. Foerster ${ }^{\mathrm{aj}}$, B. D. Fox ${ }^{\text {aj }}$,

A. Franckowiak ${ }^{\mathrm{k}}$, R. Franke ${ }^{\mathrm{am}}$, T. K. Gaisser ${ }^{\mathrm{ae}}$, J. Gallagher ${ }^{\mathrm{aa}}$, M. Geisler ${ }^{\mathrm{a}}$, L. Gerhardt ${ }^{\mathrm{h}, \mathrm{g}}$, L. Gladstone ${ }^{\mathrm{ab}}$,

T. Glüsenkamp ${ }^{\mathrm{a}}$, A. Goldschmidt ${ }^{\mathrm{h}}$, J. A. Goodman ${ }^{\mathrm{q}}$, D. Grant ${ }^{\mathrm{u}}$, T. Griesel ${ }^{\mathrm{ac}}$, A. Groß ${ }^{\mathrm{p}, \mathrm{w}}$, S. Grullon ${ }^{\mathrm{ab}}$, M. Gurtner $^{\mathrm{al}}$,

C. Ha ${ }^{\mathrm{aj}}$, A. Hallgren ${ }^{\mathrm{ak}}$, F. Halzen ${ }^{\mathrm{ab}}$, K. Han ${ }^{\mathrm{am}}$, K. Hanson ${ }^{\mathrm{m}, \mathrm{ab}}$, D. Heinen ${ }^{\mathrm{a}}$, K. Helbing ${ }^{\mathrm{a}}$, P. Herquet ${ }^{\text {ad }}$, S. Hickford ${ }^{\mathrm{p}}$,

G. C. Hill ${ }^{\text {ab }}$, K. D. Hoffman ${ }^{\mathrm{q}}$, A. Homeier ${ }^{\mathrm{k}}$, K. Hoshina ${ }^{\mathrm{ab}}$, D. Hubert ${ }^{\mathrm{n}}$, W. Huelsnitz ${ }^{\mathrm{q}}$, J.-P. Hülß ${ }^{\mathrm{a}}$, P. O. Hulth ${ }^{\text {ah }}$,

K. Hultqvist ${ }^{\text {ah }}$, S. Hussain ${ }^{\text {ae }}$, A. Ishihara ${ }^{\mathrm{o}}$, J. Jacobsen ${ }^{\mathrm{ab}}$, G. S. Japaridze ${ }^{\mathrm{d}}$, H. Johansson ${ }^{\text {ah }}$, J. M. Joseph ${ }^{\mathrm{h}}$,

K.-H. Kampert ${ }^{\mathrm{al}}$, A. Kappes ${ }^{\mathrm{i}}$, T. Karg ${ }^{\mathrm{al}, *}$, A. Karle ${ }^{\mathrm{ab}}$, J. L. Kelley ${ }^{\mathrm{ab}}$, P. Kenny ${ }^{\mathrm{z}}$, J. Kiryluk ${ }^{\mathrm{h}, \mathrm{g}}$, F. Kislat ${ }^{\mathrm{am}}$,

S. R. Klein ${ }^{\text {h,g }}$, J.-H. Köhne ${ }^{\mathrm{t}}$, G. Kohnen ${ }^{\text {ad }}$, H. Kolanoski ${ }^{\mathrm{i}}$, L. Köpke ${ }^{\text {ac }}$, S. Kopper ${ }^{\mathrm{al}}$, D. J. Koskinen ${ }^{\text {aj }}$, M. Kowalski ${ }^{\mathrm{k}}$,

T. Kowarik ${ }^{\text {ac }}$, M. Krasberg ${ }^{\text {ab }}$, T. Krings ${ }^{\text {a }}$, G. Kroll ${ }^{\text {ac }}$, K. Kuehn ${ }^{\mathrm{r}}$, T. Kuwabara ${ }^{\text {ae }}$, M. Labare ${ }^{\text {n }}$, S. Lafebre ${ }^{\text {aj }}$,

K. Laihem ${ }^{\mathrm{a}}$, H. Landsman ${ }^{\mathrm{ab}}$, M. J. Larson ${ }^{\mathrm{aj}}$, R. Lauer ${ }^{\mathrm{am}}$, J. Lünemann ${ }^{\mathrm{ac}}$, J. Madsen ${ }^{\mathrm{ag}}$, P. Majumdar ${ }^{\text {am }}$, A. Marotta ${ }^{\mathrm{m}}$,

R. Maruyama ${ }^{\mathrm{ab}}$, K. Mase ${ }^{\mathrm{o}}$, H. S. Matis ${ }^{\mathrm{h}}$, K. Meagher ${ }^{\mathrm{q}}$, M. Merck $^{\mathrm{ab}}$, P. Mészáros ${ }^{\mathrm{ai}, a j}$, T. Meures $^{\mathrm{a}}$, E. Middell ${ }^{\mathrm{am}}$,

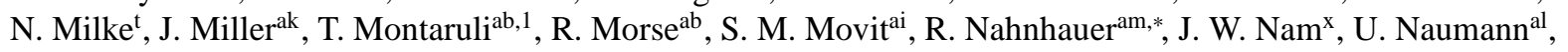

P. Nießen ${ }^{\text {ae }}$, D. R. Nygren ${ }^{\mathrm{h}}$, S. Odrowski ${ }^{\mathrm{w}}$, A. Olivas ${ }^{\mathrm{q}}$, M. Olivo ${ }^{\mathrm{j}}$, A. O’Murchadha ${ }^{\mathrm{ab}}$, M. Ono ${ }^{\mathrm{o}}$, S. Panknin ${ }^{\mathrm{k}}$,

L. Paul ${ }^{\mathrm{a}}$, C. Pérez de los Heros ${ }^{\mathrm{ak}}$, J. Petrovic ${ }^{\mathrm{m}}$, A. Piegsa ${ }^{\mathrm{ac}}$, D. Pieloth ${ }^{\mathrm{t}}$, R. Porrata ${ }^{\mathrm{g}}$, J. Posselt $^{\mathrm{al}}$, P. B. Price ${ }^{\mathrm{g}}$,

M. Prikockis ${ }^{\mathrm{aj}}$, G. T. Przybylski ${ }^{\mathrm{h}}$, K. Rawlins ${ }^{\mathrm{c}}$, P. Redl $^{\mathrm{q}}$, E. Resconi ${ }^{\mathrm{w}}$, W. Rhode ${ }^{\mathrm{t}}$, M. Ribordy $^{\mathrm{y}}$, A. Rizzo $^{\mathrm{n}}$,

J. P. Rodrigues ${ }^{\mathrm{ab}}$, P. Roth ${ }^{\mathrm{q}}$, F. Rothmaier ${ }^{\mathrm{ac}}$, C. Rott $^{\mathrm{r}}$, T. Ruhe ${ }^{\mathrm{t}}$, D. Rutledge ${ }^{\mathrm{aj}}$, B. Ruzybayev ${ }^{\mathrm{ae}}$, D. Ryckbosch ${ }^{\mathrm{v}}$,

H.-G. Sander ${ }^{\text {ac }}$, M. Santander ${ }^{\mathrm{ab}}$, S. Sarkar ${ }^{\text {af }}$, K. Schatto ${ }^{\mathrm{ac}}$, T. Schmidt ${ }^{\mathrm{q}}$, A. Schönwald ${ }^{\mathrm{am}}$, A. Schukraft ${ }^{\mathrm{a}}$,

A. Schultes ${ }^{\mathrm{al}}$, O. Schulz ${ }^{\mathrm{w}}$, M. Schunck ${ }^{\mathrm{a}}$, D. Seckel ${ }^{\mathrm{ae}}$, B. Semburg ${ }^{\mathrm{al}}$, S. H. Seo ${ }^{\mathrm{ah}}$, Y. Sestayo ${ }^{\mathrm{w}}$, S. Seunarine $^{1}$,

A. Silvestri ${ }^{\mathrm{x}}$, A. Slipak ${ }^{\mathrm{aj}}$, G. M. Spiczak ${ }^{\mathrm{ag}}$, C. Spiering ${ }^{\mathrm{am}}$, M. Stamatikos ${ }^{\mathrm{r}, 2}$, T. Stanev ${ }^{\mathrm{ae}}$, G. Stephens ${ }^{\mathrm{aj}}$,

T. Stezelberger ${ }^{\mathrm{h}}$, R. G. Stokstad ${ }^{\mathrm{h}}$, A. Stössl ${ }^{\mathrm{am}}$, S. Stoyanov ${ }^{\mathrm{ae}}$, E. A. Strahler ${ }^{\mathrm{n}}$, T. Straszheim ${ }^{\mathrm{q}}$, M. Stür ${ }^{\mathrm{k}}$,

G. W. Sullivan ${ }^{\mathrm{q}}$, Q. Swillens ${ }^{\mathrm{m}}$, H. Taavola ${ }^{\mathrm{ak}}$, I. Taboada ${ }^{\mathrm{e}}$, A. Tamburro ${ }^{\mathrm{ag}}$, A. Tepe ${ }^{\mathrm{e}}$, S. Ter-Antonyan Th $^{\mathrm{f}}$, S. Tilav $^{\mathrm{ae}}$,

P. A. Toale ${ }^{\mathrm{b}}$, S. Toscano ${ }^{\mathrm{ab}}$, D. Tosia ${ }^{\mathrm{am}}$, D. Turčan ${ }^{\mathrm{q}}$, N. van Eijndhoven ${ }^{\mathrm{n}}$, J. Vandenbroucke ${ }^{\mathrm{g}}$, A. Van Overloop ${ }^{\mathrm{v}}$,

J. van Santen ${ }^{\text {ab }}$, M. Vehring ${ }^{\mathrm{a}}$, M. Voge ${ }^{\mathrm{k}}$, C. Walck ${ }^{\text {ah }}$, T. Waldenmaier ${ }^{\mathrm{i}}$, M. Wallraff ${ }^{\mathrm{a}}$, M. Walter ${ }^{\mathrm{am}}$, Ch. Weaver $^{\text {ab }}$,

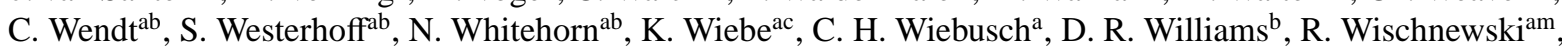

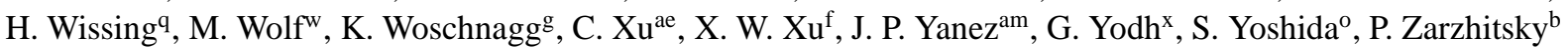

\author{
${ }^{a}$ III. Physikalisches Institut, RWTH Aachen University, D-52056 Aachen, Germany \\ ${ }^{b}$ Dept. of Physics and Astronomy, University of Alabama, Tuscaloosa, AL 35487, USA \\ ${ }^{c}$ Dept. of Physics and Astronomy, University of Alaska Anchorage, 3211 Providence Dr., Anchorage, AK 99508, USA \\ ${ }^{d}$ CTSPS, Clark-Atlanta University, Atlanta, GA 30314, USA \\ ${ }^{e}$ School of Physics and Center for Relativistic Astrophysics, Georgia Institute of Technology, Atlanta, GA 30332, USA \\ ${ }^{f}$ Dept. of Physics, Southern University, Baton Rouge, LA 70813, USA \\ ${ }^{g}$ Dept. of Physics, University of California, Berkeley, CA 94720, USA \\ ${ }^{h}$ Lawrence Berkeley National Laboratory, Berkeley, CA 94720, USA \\ institut für Physik, Humboldt-Universität zu Berlin, D-12489 Berlin, Germany \\ ${ }^{j}$ Fakultät für Physik E Astronomie, Ruhr-Universität Bochum, D-44780 Bochum, Germany
}

\footnotetext{
${ }^{*}$ Corresponding authors

Email addresses: jens.berdermann@desy.de (J. Berdermann), karg@uni-wuppertal.de (T. Karg), rolf .nahnhauer@desy.de (R. Nahnhauer)

${ }^{1}$ also Università di Bari and Sezione INFN, Dipartimento di Fisica, I-70126, Bari, Italy

${ }^{2}$ NASA Goddard Space Flight Center, Greenbelt, MD 20771, USA
} 


\author{
${ }^{k}$ Physikalisches Institut, Universität Bonn, Nussallee 12, D-53115 Bonn, Germany \\ ${ }^{l}$ Dept. of Physics, University of the West Indies, Cave Hill Campus, Bridgetown BB11000, Barbados \\ ${ }^{m}$ Université Libre de Bruxelles, Science Faculty CP230, B-1050 Brussels, Belgium \\ ${ }^{n}$ Vrije Universiteit Brussel, Dienst ELEM, B-1050 Brussels, Belgium \\ ${ }^{o}$ Dept. of Physics, Chiba University, Chiba 263-8522, Japan \\ ${ }^{p}$ Dept. of Physics and Astronomy, University of Canterbury, Private Bag 4800, Christchurch, New Zealand \\ ${ }^{q}$ Dept. of Physics, University of Maryland, College Park, MD 20742, USA \\ ${ }^{r}$ Dept. of Physics and Center for Cosmology and Astro-Particle Physics, Ohio State University, Columbus, OH 43210, USA \\ ${ }^{s}$ Dept. of Astronomy, Ohio State University, Columbus, OH 43210, USA \\ ${ }^{t}$ Dept. of Physics, TU Dortmund University, D-44221 Dortmund, Germany \\ ${ }^{u}$ Dept. of Physics, University of Alberta, Edmonton, Alberta, Canada T6G 267 \\ ${ }^{v}$ Dept. of Physics and Astronomy, University of Gent, B-9000 Gent, Belgium \\ ${ }^{w}$ Max-Planck-Institut für Kernphysik, D-69177 Heidelberg, Germany \\ ${ }^{x}$ Dept. of Physics and Astronomy, University of California, Irvine, CA 92697, USA \\ ${ }^{y}$ Laboratory for High Energy Physics, École Polytechnique Fédérale, CH-1015 Lausanne, Switzerland \\ ${ }^{z}$ Dept. of Physics and Astronomy, University of Kansas, Lawrence, KS 66045, USA \\ ${ }^{a a}$ Dept. of Astronomy, University of Wisconsin, Madison, WI 53706, USA \\ ${ }^{a b}$ Dept. of Physics, University of Wisconsin, Madison, WI 53706, USA \\ ${ }^{a c}$ Institute of Physics, University of Mainz, Staudinger Weg 7, D-55099 Mainz, Germany \\ ${ }^{a d}$ Université de Mons, 7000 Mons, Belgium \\ ${ }^{a e}$ Bartol Research Institute and Department of Physics and Astronomy, University of Delaware, Newark, DE 19716, USA \\ ${ }^{a f}$ Dept. of Physics, University of Oxford, 1 Keble Road, Oxford OX1 3NP, UK \\ ${ }^{a g}$ Dept. of Physics, University of Wisconsin, River Falls, WI 54022, USA \\ ${ }^{a h}$ Oskar Klein Centre and Dept. of Physics, Stockholm University, SE-10691 Stockholm, Sweden \\ ${ }^{a i}$ Dept. of Astronomy and Astrophysics, Pennsylvania State University, University Park, PA 16802, USA \\ ${ }^{a j}$ Dept. of Physics, Pennsylvania State University, University Park, PA 16802, USA \\ ${ }^{a k}$ Dept. of Physics and Astronomy, Uppsala University, Box 516, S-75120 Uppsala, Sweden \\ ${ }^{a l}$ Dept. of Physics, University of Wuppertal, D-42119 Wuppertal, Germany \\ ${ }^{\text {am } D E S Y, ~ D-15735 ~ Z e u t h e n, ~ G e r m a n y ~}$
}

\begin{abstract}
The detection of acoustic signals from ultra-high energy neutrino interactions is a promising method to measure the flux of cosmogenic neutrinos expected on Earth. The energy threshold for this process depends strongly on the absolute noise level in the target material. The South Pole Acoustic Test Setup (SPATS), deployed in the upper part of four boreholes of the IceCube Neutrino Observatory, has monitored the noise in Antarctic ice at the geographic South Pole for more than two years down to $500 \mathrm{~m}$ depth. The noise is very stable and Gaussian distributed. Lacking an in-situ calibration up to now, laboratory measurements have been used to estimate the absolute noise level in the 10 to $50 \mathrm{kHz}$ frequency range to be smaller than $20 \mathrm{mPa}$. Using a threshold trigger, sensors of the South Pole Acoustic Test Setup registered acoustic events in the IceCube detector volume and its vicinity. Acoustic signals from refreezing IceCube holes and from anthropogenic sources have been used to test the localization of acoustic events. An upper limit on the neutrino flux at energies $E_{v}>10^{11} \mathrm{GeV}$ is derived from acoustic data taken over eight months.
\end{abstract}

Keywords: acoustic neutrino detection, absolute noise level, neutrino flux limit

PACS: 43.58.+z, 43.60.+d, 93.30.Ca

\section{Introduction}

During recent years it has been extensively studied, whether the glacier ice at the South Pole is a suitable material for the detection of cosmic neutrinos above $10^{18} \mathrm{eV}$ energy, using their acoustic signals emitted in the 10 to $50 \mathrm{kHz}$ region. With data from the South Pole Acoustic Test Setup [1], the speed of sound has been measured down to $500 \mathrm{~m}$ depth. It was found to be con- stant below $200 \mathrm{~m}$, favorable for neutrino detection [2]. The sound attenuation in the 200 to $500 \mathrm{~m}$ depth region was measured to be much stronger than expected [3]. An important precondition for acoustic neutrino detection is a precise knowledge of the steady and transient noise levels on top of which a possible neutrino signal has to be detected.

Long duration noise studies in deep water of the Mediterranean Sea [4, 5] and Lake Baikal [6] have 
shown strong variations with time due to changing environmental conditions such as wind speed and rain. Sound sources such as ships and animals (e.g. sperm whales) contribute as well. In quiet periods, however, noise levels as low as a few $\mathrm{mPa}$ are reported for the frequency region of interest.

Acoustic noise at the South Pole is assumed to be low and stable because none of the sources mentioned above are expected to contribute. Due to the depth dependence of the density, the first $200 \mathrm{~m}$ of firn ice acts as an acoustic filter. Anthropogenic and environmental noise entering the ice from the surface will be refracted back.

The glacier moves with a velocity of about $10 \mathrm{~m}$ per year over the bedrock beneath. Whether this or other phenomena give rise to ultra-sound of detectable amplitude is one subject of the investigations presented in this paper.

In Sec. 2, the detector geometry, calibration and the various data taking modes will be described. In Sec. 3 . the permanent noise conditions will be discussed, followed by information about the detection of sound signals produced in the ice by construction activities of the IceCube experiment [7] in Sec. 4, Finally, a limit for the flux of cosmic neutrinos will be derived from acoustic data accumulated during eight months.

\section{Detector setup and data taking}

\subsection{Detector geometry}

To measure the acoustic properties of the ice at the geographic South Pole, the South Pole Acoustic Test Setup (SPATS) [1], a system of four instrumented vertical lines, called strings A, B, C, and D, was installed in boreholes of the IceCube neutrino observatory after deployment of the IceCube optical modules. Each string holds seven stages, referred to as stages 1 to 7 from top to bottom; each stage is a combination of an acoustic sensor and a transmitter, vertically separated by $\sim 1 \mathrm{~m}$. The horizontal distances between strings range from $125 \mathrm{~m}$ (which is the spacing between IceCube strings) to $543 \mathrm{~m}$. Vertically, the depth range from 80 to $500 \mathrm{~m}$ is instrumented with increasing spacing of sensors in the deeper ice to be able to sample the transition from the firn 3 region to the bulk ice. On strings A, $\mathrm{B}$, and C the stages are located at depths of 80, 100, 140, 190, 250, 320, and $400 \mathrm{~m}$; string D is deployed deeper in the ice and the stages are located at 140, 190, 250, $320,400,430$, and $500 \mathrm{~m}$ depth.

\footnotetext{
${ }^{3}$ The transition region from a snow/air mixture at the surface to solid ice is called firn. It has a width of about $170 \mathrm{~m}$ at South Pole [2].
}

The SPATS sensors consist of a cylindrical stainless steel pressure housing with a diameter of $10 \mathrm{~cm}$ in which three piezoelectric elements are mounted to the wall, separated by 120 degrees to provide full azimuthal coverage. A three-stage low noise pre-amplifier with a gain of $10^{4}$ is attached directly to each piezoelectric element and the analog signal is transmitted to the surface via a shielded twisted pair cable. On string D at depths of $190 \mathrm{~m}$ and $430 \mathrm{~m}$, an alternative type of sensor, HADES (Hydrophone for Acoustic Detection at South Pole), was deployed. For the HADES sensors, the piezoelectric element and pre-amplifier have been cast in resin and mounted below the housing. This allows us to study acoustic signals with different systematics introduced by the sensor.

The sensor channels are each identified by a label of the form $X \operatorname{Sy} y(z)$, where $X$ is the letter of the string, $\mathrm{S}$ refers to a sensor, $y$ is the number of the stage, and $z$ the channel number within the sensor (running from 0 to 2 ).

\subsection{Data acquisition}

On the surface, a read-out box buried in the snow above each string contains an industrial PC, called string-PC, that is used for digitization, time stamping, and storage of the data. Each string-PC is connected by a symmetric DSL connection to the SPATS master-PC that is housed in the IceCube laboratory at the South Pole station. The master-PC collects the data from all four string-PCs, distributes a GPS timing signal to them, and prepares the data for transfer to the northern hemisphere via satellite or for tape storage.

Since 28 August 2008, SPATS has been operating as a detector for transient acoustic signals. Out of each hour, 45 minutes are used for triggered data taking while in the remaining 15 minutes environmental monitoring and system health data are recorded, including measurements of the noise floor. Details on the DAQ system can be found in Ref. [1]. To monitor the noise floor, every hour the three channels of each sensor simultaneously record $100 \mathrm{~ms}$ of untriggered data with a sampling rate of $200 \mathrm{kHz}$. In transient data taking mode, three sensor channels from each string are used, located at three different depths. The data stream from each channel, digitized with a sampling rate of $200 \mathrm{kHz}$, is continuously monitored in the string-PC. A sensor noise band is defined for each channel $i$ spanning symmetrically around the channels noise mean value $\mu_{i}$ from $\mu_{i}-5.2 \sigma_{i}$ to $\mu_{i}+5.2 \sigma_{i}$ as illustrated in Fig. 2, where $\sigma_{i}$ is the width of the noise distribution of the respective channel. If an ADC value $x_{i}$ outside the noise band $\left(x_{i}>\mu_{i}+5.2 \sigma_{i}\right.$ or $x_{i}<\mu_{i}-5.2 \sigma_{i}$ is measured, $5 \mathrm{~ms}$ of data (1001 samples) are written to disk, centered on the triggering sample. 
The threshold of $5.2 \sigma_{i}$ was chosen to fit the amount of data into the allocated daily satellite bandwidth of $150 \mathrm{MB}$. The detector has been operated in two different modes, hereafter called mode 1 and mode 2 . In mode 1 , the sensors located at 190, 250, and $320 \mathrm{~m}$ depth were used for transient data taking. In mode 2 a deeper sensor configuration $(250,320,400 \mathrm{~m})$ was chosen.

One transient data file per string is written every hour. Transient data taking runs are successful in $93 \%$ of all cases. The remaining runs commonly fail due to DAQ problems. No correlation between failing runs and large amplitude pulses, which would bias the analysis described in this work, has been observed. During measurements with the acoustic "pinger" [2, 3] large amplitude pulses have been recorded with a rate of up to $10 \mathrm{~Hz}$ without an increase in the occurrence of failed runs.

As can be seen in Fig. 1(a) there are quiet periods during the austral winter and more noisy periods during summer with IceCube hole drilling and string deployment taking place. We distinguish between four periods of data taking; details can be found in Tab. 1. On average, about 65000 triggers are collected per day. The data are dominated by sensor noise, i.e. triggers caused by single ADC samples from the tails of the Gaussian noise distribution. In the quiet periods, these account for $99.99 \%$ of all recorded waveforms, for which no coincident signals in other sensor channels are observed. Nearly all of these triggers do not contain typical waveforms but have only a single spike larger than the trigger threshold.

\subsection{Sensor calibration}

The sensitivity 4 and equivalent self-noise 5 of all SPATS sensors were measured in the laboratory prior to deployment using the comparison method. The mean sensitivity (including the pre-amplifier) averaged over all sensor channels and all frequencies in the relevant frequency band from 10 to $50 \mathrm{kHz}$ is $2.8 \pm 0.8 \mathrm{~V} \mathrm{~Pa}^{-1}$ (equal to $-111 \mathrm{~dB}$ re. $1 \mathrm{~V} \mu \mathrm{Pa}^{-1}$ ), where the error indicates the spread between the different sensors. The equivalent self-noise in the same frequency band, averaged over all SPATS sensors, is $7 \mathrm{mPa}$. Details of the measurement are presented in Ref. [1]. The calibration was performed in water at a temperature of $0^{\circ} \mathrm{C}$ and at

\footnotetext{
${ }^{4}$ Sensitivity is defined as the ratio $V / p$ of the voltage $V$ induced at the output of an acoustic sensor, given a pressure amplitude $p$, with units of $\mathrm{V} \mathrm{Pa}^{-1}$.

${ }^{5}$ Equivalent self-noise is defined as the electronic self-noise of the device converted into an equivalent sound pressure level using the sensor's sensitivity.
}

normal pressure. It is not clear a priori, whether the sensitivity of the sensors remains unchanged when deployed deep in Antarctic ice at a temperature of $-50^{\circ} \mathrm{C}$ and an increased but not well known static ambient pressure. The water in the IceCube boreholes starts to freeze from the top and from the sides so that cavities are formed in which the static pressure will increase above the pressure of the water column at sensor depth. After the hole is completely frozen, relaxation of the freshly frozen "hole ice" to the surrounding bulk ice occurs on an unknown time scale. Furthermore, the sensitivity of the sensor can be influenced by the different acoustic coupling, determined by the different acoustic impedance matching between the water-sensor interface and the ice-sensor interface, by the unknown horizontal position of the sensor within the IceCube hole, and by possible shadowing of parts of the sensor by the IceCube main cable running down the hole parallel to the sensors.

Due to the absence of standardized acoustic sources for ice, it is not possible to recalibrate the sensors after deployment. Also, it is beyond the scope of the SPATS project to reproduce the combined influence of very low temperatures, high static pressure and ice in a laboratory environment. However, we can study the influence of these aspects on the sensitivity by separate investigations. In the following discussion, we will assume that the influences of the environmental parameters are uncorrelated and can thus be used to estimate the in-situ sensor sensitivity from the results of different laboratory experiments. A typical SPATS sensor was calibrated in air at different temperatures. A linear increase of its sensitivity with decreasing temperature was measured. The sensitivity increases by a factor of $1.5 \pm 0.2$ from $0^{\circ} \mathrm{C}$ to $-50^{\circ} \mathrm{C}[1]$. The same sensor was calibrated at room temperature in a water filled pressure vessel at different static pressures from 1 bar to 100 bar. No systematic change in the sensitivity with pressure was observed; the sensitivity was found to be stable within 30\% [1]. The effect of the acoustic coupling of the sensor to the ice, which can lead to a flattening of the frequency response function and a reduction of the sensitivity due to a damping of mechanical resonances of the sensor housing, is not yet understood and will be studied in an ice block in the laboratory using the reciprocity calibration method (for a discussion of the reciprocity calibration method, see e.g. [8]).

Since the correlation between the different environmental parameters is unknown when the sensor is exposed to a combination of all of them in the deep Antarctic ice, the determination of the absolute noise level is currently only roughly possible. Multiplying the 


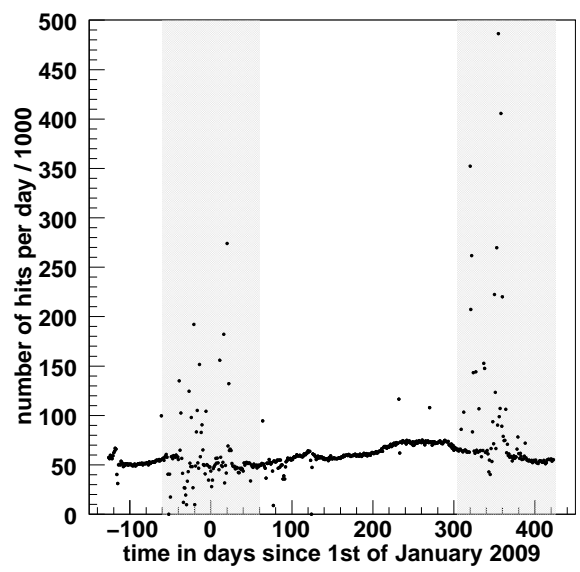

(a)

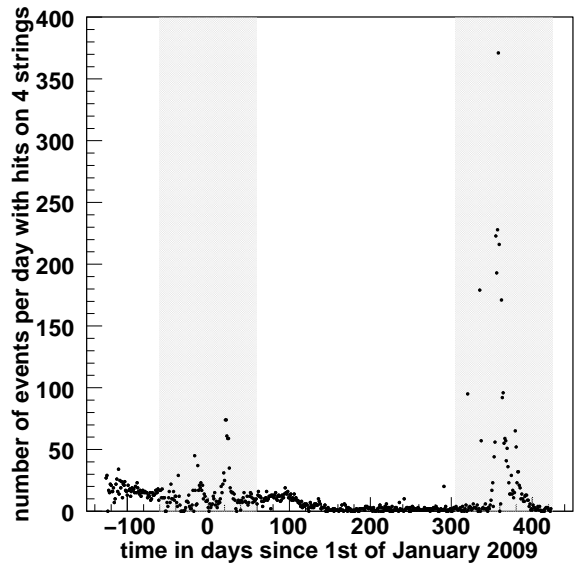

(b)

Figure 1: (a) Number of acoustic hits per day and (b) acoustic events with hits at all 4 strings since 1 January 2009 . Drill periods are indicated in light gray. For the definition of a "hit" and an "event" see Sec.4

Table 1: Characteristics for different data taking periods of transient noise triggers

\begin{tabular}{|l|r|r|r|r|}
\hline Name & Quiet period 1 & Drill period 1 & Quiet period 2 & Drill period 2 \\
\hline \hline Start date & 28 Aug. 2008 & 1 Nov. 2008 & 1 Mar. 2009 & 1 Nov. 2009 \\
\hline Duration (days) & 65 & 120 & 245 & 120 \\
\hline Available files & 5820 & 9845 & 22664 & 10567 \\
\hline Avail./total & 0.93 & 0.85 & 0.96 & 0.92 \\
\hline Detector mode & 1 & 1 & 2 & 2 \\
\hline
\end{tabular}


sensor's sensitivity changes due to temperature $(\times 1.5)$ and due to pressure $(\times 1)$ and adding the uncertainties in quadrature, we find that the sensitivity of the sensor in the deep ice is increased by a factor of $1.5 \pm 0.4$ as compared to the value obtained in the laboratory, resulting in a mean sensitivity of $4.2 \pm 1.6 \mathrm{~V} \mathrm{~Pa}^{-1}$.

\section{Properties of the noise floor}

\subsection{General}

We observe a Gaussian distribution of ADC values for each sensor channel. Thus the noise can be characterized by two parameters: a mean value and a standard deviation. The mean value depends on an instrumental DC offset in the different channels and is always close to zero. The standard deviation is a measure for the noise level in the sensor, which is a superposition of sensor electronic self-noise, electromagnetic interference picked up on the signal cable from the sensor to the surface 6 , and possible acoustic noise contributions from the surrounding ice.

The distribution of ADC values from $73.1 \mathrm{~s}$ of recorded noise data is shown in Fig. 22 The dynamic range of the 12-bit $\mathrm{ADC}$ is $\pm 5 \mathrm{~V}$, corresponding to $2.4 \mathrm{mV}$ per ADC count. The data are perfectly described by a Gaussian. The mean value and trigger thresholds for transient data taking are indicated in the graph. The four samples outside the noise band match very well the expectation from the average SPATS trigger rate of 4.6 triggers during these $73.1 \mathrm{~s}$.

\subsection{Stability}

We have monitored the noise level in all sensor channels for more than three years, beginning with the deployment of the first SPATS sensors in January 2007. Figure 3 shows the RMS of the noise as a function of time for three typical sensor channels on string $C$ that participate in the transient data taking. All available data from deployment until autumn 2010 is shown. It can be seen that, apart from some short-time excesses that will be discussed below, the noise level is very stable, the typical fluctuations being $\sigma_{\mathrm{RMS}} /\langle\mathrm{RMS}\rangle\left\langle 10^{-2}\right.$.

In 2007, the first year of SPATS operation, we measured a higher and less stable noise level. During this period, the sensors were powered on only for data taking and powered off afterwards which causes them to

\footnotetext{
${ }^{6}$ Electromagnetic interference is expected to be small since the signal is transmitted differentially from the sensor to the ADC on the surface.
}

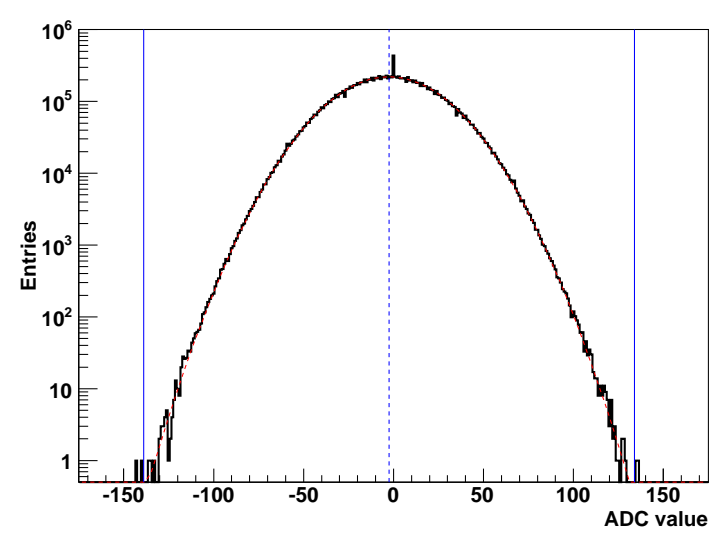

Figure 2: Distribution of ADC values from all noise data recorded for channel CS7(2) in July 2009 (73.1 s of data in total). The red dashed line is a Gaussian fit to the data; the vertical lines indicate the mean value $\mu$ (dashed) and the trigger thresholds at $\mu \pm 5.2 \sigma$. The peak at ADC value 0 is an understood feature of the ADC.

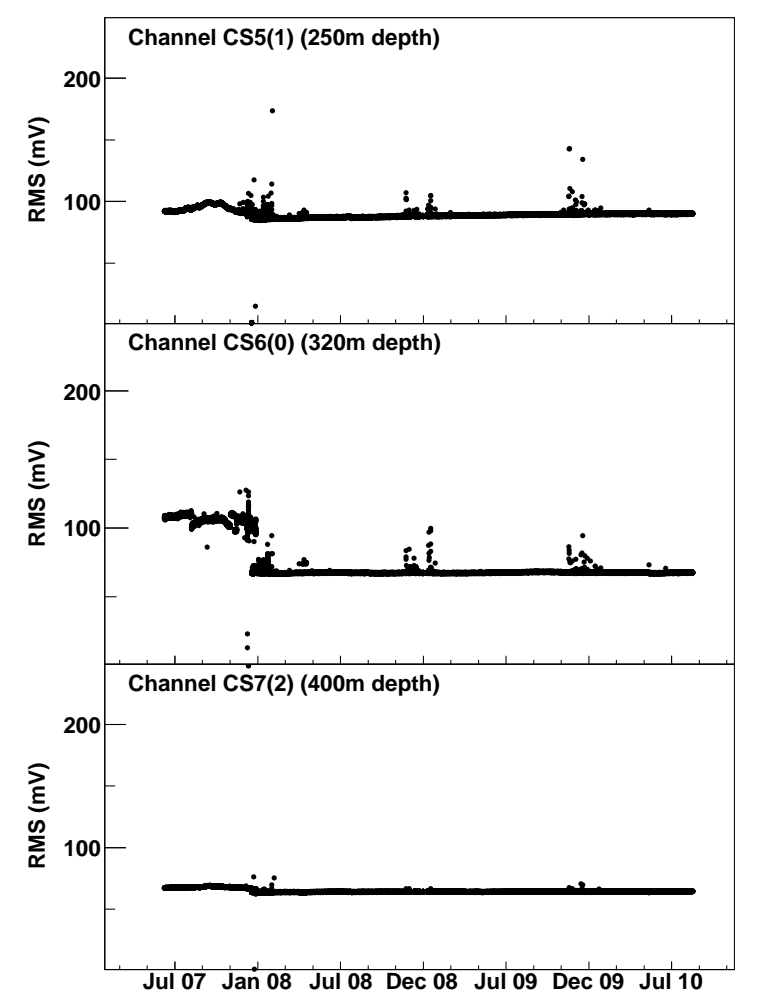

Figure 3: Noise RMS (from zero to $100 \mathrm{kHz}$ ) as a function of time for three sensors on string $\mathrm{C}$ participating in transient data taking. The time window spans all data from deployment till today. 


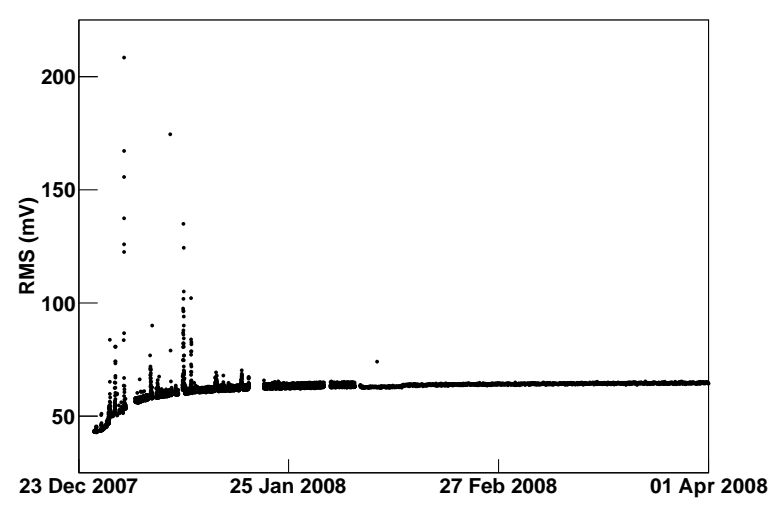

Figure 4: Noise RMS as a function of time during freeze in for sensor channel DS7(2) deployed at $500 \mathrm{~m}$ depth.

heat up during the measurement and change their selfnoise characteristics. Since December 2007, all sensors are powered on continuously and are in thermal equilibrium with the surrounding ice. Noise studies following a power outage show that it takes several hours for a sensor to reach thermal equilibrium after it is powered on. The short-term noise excesses, which occur only in the Austral summer seasons, can be correlated to IceCube deep-ice drilling activity. The visible spikes correspond to the holes drilled closest to the SPATS array. Due to technical reasons, data on the noise level during the freeze in of the sensors after deployment is only available for string D which was installed one year after the other three strings. It is shown for one sensor channel in Fig. 4

We observe an increase of the noise level after the deployment of the sensor on 24 December 2007 that lasts for about three weeks, after which the noise level became stable. On top of that, excesses correlated with IceCube deep-ice drilling can be seen. We interpret the rise of the noise level as a combination of the increase of sensitivity with decreasing temperature (cf. Sec. 2.3) and an improved acoustic coupling of the sensor to the bulk ice.

\subsection{Determination of the absolute noise level}

To determine the absolute noise level from the data, i.e. the sound pressure incident on the sensor, the sensitivity needs to be known. In general, the sensitivity will be a function of direction and frequency. In the absence of a specific noise source model, an equivalent acoustic power at the position of the piezoelectric element is derived assuming isotropic noise. The power is then translated into an effective pressure amplitude.

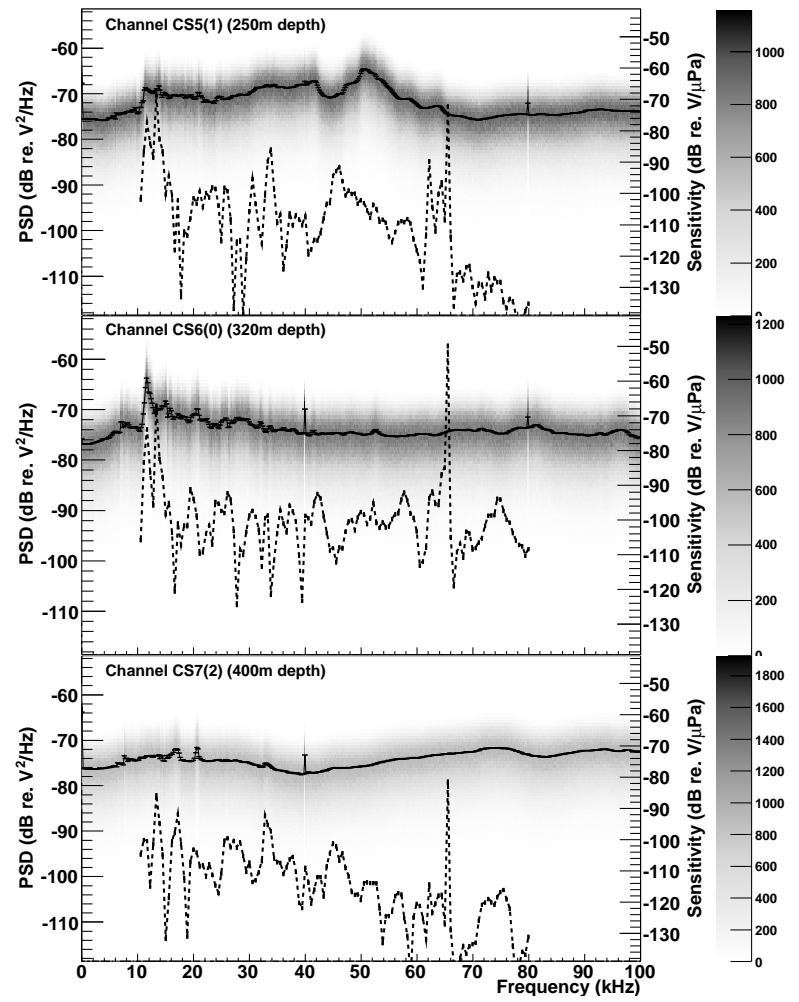

Figure 5: Average power spectral density (PSD, shown as solid lines) and distribution for each frequency bin (gray scales) for three sensors on string $\mathrm{C}$ participating in transient data taking. All noise data recorded in July 2010 are shown. For comparison, the sensitivity of the sensor channels measured in the laboratory prior to deployment is shown as dashed lines.

In Fig. 5, we show the voltage power spectral density (PSD) distribution for three sensor channels participating in transient data taking. The plot was obtained as follows: all noise floor data recorded in July 2010 were Fourier transformed in sets of 1000 samples each (frequency resolution $\Delta f=200 \mathrm{~Hz}$ ) and the corresponding PSD values were filled into a two dimensional histogram. The gray scale is a measure for the probability of occurrence of a certain PSD value at a given frequency. The solid lines represent the mean values, calculated on a linear PSD scale, in each frequency bin. The error of the mean is also indicated, but too small to be visible.

Figure 5 demonstrates that the spectral shapes differ between all sensors. The sensitivity of the sensors as a function of frequency, as measured in water in the laboratory prior to deployment, is indicated as dashed lines. It is expected that the resonance structure of the PSD is mainly governed by the mechanical response of the sensor and not determined by the spectrum of the incident 
acoustic background noise on the sensor. Especially the steel housing and its coupling to the piezoelectric ceramic, which can be slightly different for different sensors, should have a large effect. It can be seen in Fig. 5 that peaks in the sensitivity are not reflected as peaks in the PSD as would be expected for a smooth acoustic noise spectrum. This supports the assumption that the resonance behavior of the sensor, and thus its sensitivity, is modified by the coupling of the sensor housing to the ice. Due to the suspected change in the spectral sensitivity during freeze-in, we will not calculate the absolute noise level by dividing the PSD by the sensitivity to determine the noise spectrum in units of pressure density and integrate over the relevant frequency range. This procedure would introduce unknown errors by underestimating the contribution from frequency regions with high sensitivity in the laboratory calibration. Instead, we assume a single mean sensitivity for all sensors, determined by averaging the laboratory sensitivity of each sensor over the frequency range from 10 to $50 \mathrm{kHz}$ and subsequently averaging over all sensors and applying the correction factor for temperature and pressure. This procedure yields a mean sensitivity of $\left\langle S_{10-50}\right\rangle=4.2 \pm 1.6 \mathrm{~V} \mathrm{~Pa}^{-1}$ as discussed in Section 2.3 We determine the absolute noise level from each sensors voltage PSD integrated from 10 to $50 \mathrm{kHz}$, using the July 2010 data presented in Fig. 5. Attenuation losses in the cable of $-0.6 \mathrm{~dB} / 100 \mathrm{~m}$ are corrected for. This assumes the worst case scenario that all the measured noise is produced in the sensor or is acoustic noise in the ice and no additional electromagnetic noise is induced during transmission. Noise induced further upstream in the DAQ chain would be over-corrected for cable attenuation and result in an overestimation of the noise. Figure 6 shows the resulting noise level for all operative SPATS channels. We separate the sensors into two groups: sensors above $200 \mathrm{~m}$ depth and sensors below $200 \mathrm{~m}$. The latter ones are used for the transient noise analysis in the remainder of this work. For the shallow sensors, we calculate a mean noise level of $21 \mathrm{mPa}$ with a $5 \mathrm{mPa}(1 \sigma)$ spread between the data points. The average noise level in the deep sensors is $(16 \pm 3) \mathrm{mPa}$. This still includes the contribution from electronic self-noise, that has been measured in the laboratory prior to deployment to be $7 \mathrm{mPa}$ equivalent on average. Subtracting this contribution quadratically leads to an estimated mean noise level in South Polar ice of $20 \mathrm{mPa}$ (shallow) and $14 \mathrm{mPa}$ (deep) integrated over the frequency range relevant for acoustic neutrino detection of 10 to $50 \mathrm{kHz}$. Using the simulation described in Sec. 5 and assuming an acoustic attenuation length of $300 \mathrm{~m}$, a pressure of $14 \mathrm{mPa}$ corresponds to the amplitude of the acoustic sig-

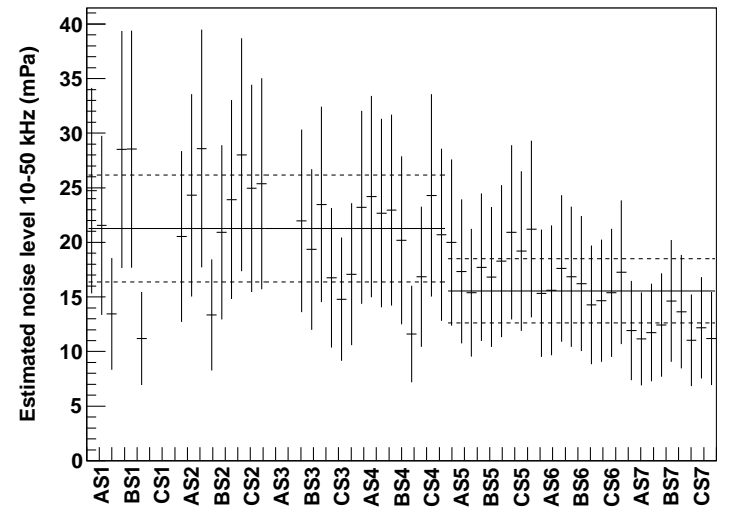

Figure 6: Estimated absolute noise level integrated from 10 to $50 \mathrm{kHz}$ for all SPATS channels. The error bars indicate the uncertainty on the sensitivity of the sensor channels. Sensors above $200 \mathrm{~m}$ depth (stages 1 to 4 ) and below $200 \mathrm{~m}$ (stages 5 to 7 ) are treated separately (see text for details). Sensors AS3 and CS1 are broken and no data are available. The solid lines indicate the mean values and the dashed lines indicate the $1 \sigma$ spread of the data points. An average equivalent self-noise of $7 \mathrm{mPa}$ can be subtracted quadratically for all sensors.

nal generated by a neutrino of energy $10^{11} \mathrm{GeV}$ interacting in a distance of about $1000 \mathrm{~m}$.

The origin and significance of the decrease of the noise level with depth, that is visible in Fig. 6, remains unclear. One possible qualitative explanation for the observed depth dependence is a contribution of noise generated on the surface. Due to the gradient in the sound speed with depth [2], all noise from the surface will be refracted back towards the surface, thus shielding deeper regions from surface noise.

\section{Transient noise events}

A triggered waveform ("hit") contains 500 samples measured before the trigger and 500 samples after the trigger, each separated by $5 \mu \mathrm{s}$ (see also Sec. 2.2). If the acoustic signal lasts longer than $2.5 \mathrm{~ms}$, the following trigger is added to the hit under consideration. Hits from all strings are ordered in time offline and merged into one file per day. This file is processed through a cluster algorithm to find all consecutive hits within $200 \mathrm{~ms}$, the time necessary for an acoustic signal to cross the SPATS array. All hits per cluster are considered to form an acoustic event. Events with more than 5 hits from at least 3 strings are used to localize their source position in the ice (see Section 4.1). The distribution in time of acoustic events with hits from all four strings is shown in Fig. 1(b) 


\subsection{Source vertex reconstruction}

The acoustic event reconstruction algorithm is based on the solution of the system of equations with $n=$ $1, \ldots, 4$

$\left(x_{n}-x_{0}\right)^{2}+\left(y_{n}-y_{0}\right)^{2}+\left(z_{n}-z_{0}\right)^{2}=\left[v_{s}\left(t_{n}-t_{0}\right)\right]^{2}$.

Only four sensors with their signal arrival times and positions $t_{n}, x_{n}, y_{n}, z_{n}$ are used in a single reconstruction. The calculated event vertex is located at the space time point $t_{0}, x_{0}, y_{0}, z_{0}$, where the $\mathrm{z}$-axis points vertically upward and $z=0$ corresponds to the ice surface. The signal velocity in ice is taken to be constant with $v_{s}=3878 \mathrm{~m} / \mathrm{s}$ [2], and the propagation direction is assumed to be straight. The assumption of a constant speed of sound is only suitable for events below a depth of around $200 \mathrm{~m}$ and leads to a spread of reconstructed event positions for shallower depths, as one can see from simulations (Section 4.3). Solving the system of equations above provides an event vertex for a single four sensor combination. With twelve sensors in the used SPATS configuration, statistical predictions can be made by using all possible combinations $i=1, \ldots, m$ of four sensors on four different strings per acoustic event. In case of a noise hit in a sensor, the reconstruction algorithm for this combination does not converge or the result lies far outside the sensitive SPATS area. The deviation from the mean vertex position of all possible sensor combinations

$$
\overline{\vec{r}}=\frac{1}{m} \sum_{i=1}^{m} \vec{r}_{i},
$$

with $\vec{r}_{i}=\left(x_{0}^{i}, y_{0}^{i}, z_{0}^{i}\right)$, is used to improve upon the background rejection. This is done by rejecting reconstruction results for a single sensor combination if the distance from the mean position in $x, y$ or $z$ is above $250 \mathrm{~m}$.

\subsection{Acoustic event sources}

In Fig. 7(a), all reconstructed four-string events are plotted according to their position in the IceCube coordinate frame. Figure 7(b) shows their location with respect to the SPATS strings (large circles) and IceCube holes (small circles). The triangles reflect the positions at which a Rodriguez-Well (RW for short) [10] is located (see Table 2). Such wells are used for the production and cycling of water for the IceCube hot water drill system. As can be seen, the acoustic events are concentrated either at IceCube boreholes or at Rodriguez-Well locations. In Fig. 7(c), the event depth distribution versus time is shown. Almost no events are located above $50 \mathrm{~m}$ depth. In quiet periods, events are concentrated between 80 and $150 \mathrm{~m}$. During drilling periods vertices are still found down to $600 \mathrm{~m}$.

\subsubsection{Events from IceCube holes}

Acoustic events were observed from nearly all IceCube holes drilled in both seasons, when transient data taking was active.

The statistics collected in the second season was much larger, due to the fact that most of the 2009/10 holes were located close to the center of the SPATSdetector. The event distributions derived from different holes are very similar. We investigate 2093 events from hole 81 , the hole with the highest statistic, as an example. Events are observed for 20 days in the hole region ( $\pm 20 \mathrm{~m}$ with respect to the center of hole 81$)$ during the periods of firn ice drilling $(<50 \mathrm{~m}$ depth), bulk ice drilling (50 to $2500 \mathrm{~m}$ depth) and refreezing, as can be seen in Fig. 8(a). Figure 8(b) shows the depth distribution of the events versus time. Before drilling, events are observed at 40 to $100 \mathrm{~m}$ depth probably connected with noise from the firn drill hole. During the procedure of hot water drilling a few related events are found. Strong sound production starts about three days after drilling is finished, due to the refreezing process. About $30 \%$ of the registered events from this hole are concentrated in two spots at $120 \mathrm{~m}$ and $250 \mathrm{~m}$ depth but reach down to about $600 \mathrm{~m}$. The reason is that the hole does not refreeze homogeneously, but forms frozen ice plugs between regions that are still filled with water. The pressure produced in this way may give rise to cracks near the ice water boundary which would appear with sound in the 10 to $100 \mathrm{kHz}$ frequency region. Relaxation later continues within "arms" freezing towards the hole surface and down to the lower ice plug (see Fig. 8(b)). Besides providing information about the refreezing process of water filled IceCube holes, one can also use the corresponding acoustic events to understand the precision of the vertex localization algorithm. In Fig. 9(a) and Fig. 9(b) the reconstructed $x$ and $y$ event positions are shown for hole 81 in the IceCube reference coordinate system. The average values including statistical uncertainties for the $(x, y)$ position of hole 81 are determined to $(42.0 \pm 0.1) \mathrm{m}$ in $x$ and $(38.5 \pm 0.1) \mathrm{m}$ in $y$. The width of the distributions is $2.6 \mathrm{~m}$ and $5.0 \mathrm{~m}$ respectively, to be compared with a hole diameter of about $0.7 \mathrm{~m}$. The calculated values deviate from the actual hole positions at the surface by $0.4 \mathrm{~m}$ (in $x$ ) and $3.0 \mathrm{~m}$ (in $y$. 7 . The possible reason for this deviation will be discussed in the simulation section (Sec.4.3) below.

\footnotetext{
${ }^{7}$ The actual positions of the sensors in the $x-y$ plane in the holes are known with a precision of $0.5 \mathrm{~m}$ due to the hole width and inclinations versus depth from the drilling process.
} 


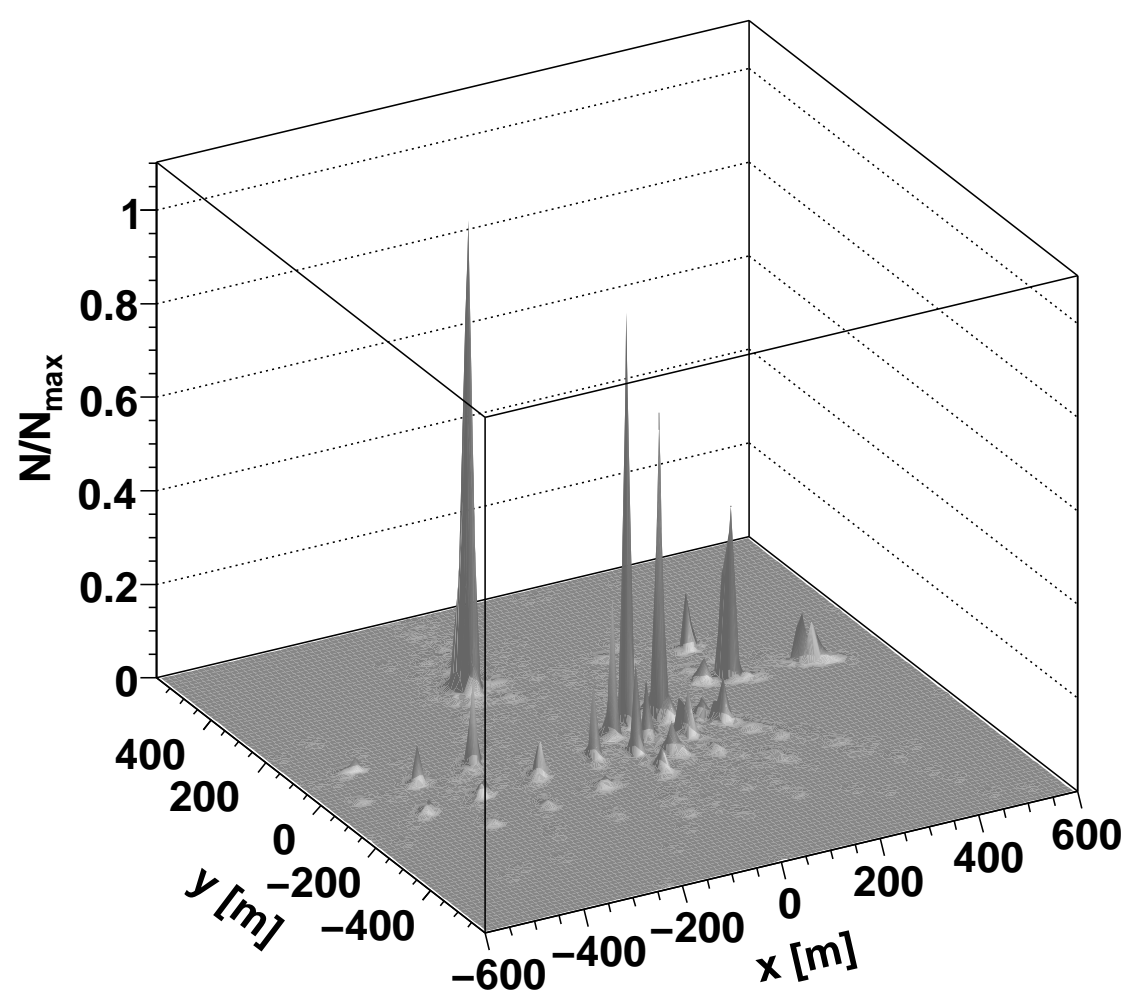

(a)

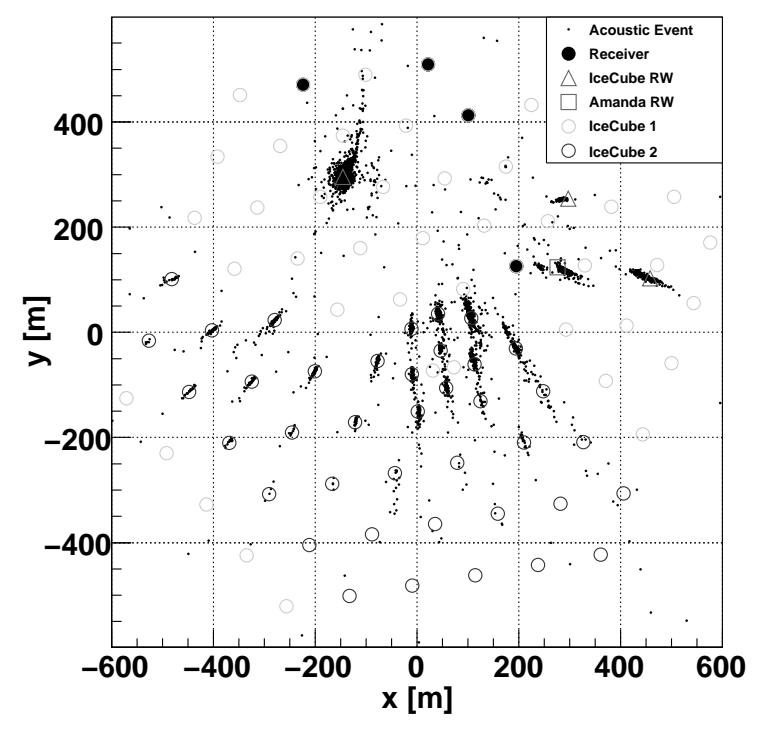

(b)

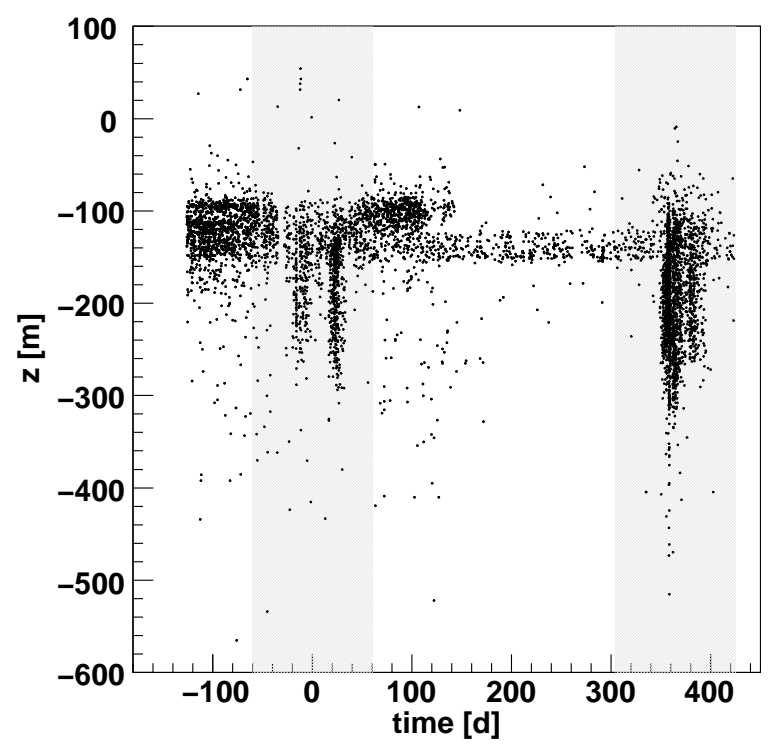

(c)

Figure 7: Shown are (a) the relative abundance of reconstructed acoustic events in the horizontal plane of the IceCube coordinate system and (b) the actual vertex position of all transient events recorded since August 2008. The sources of transient noise are the Rodriguez Wells (RW), large caverns melted in the ice for water storage during IceCube drilling, and the refreezing IceCube holes. Dark gray circles (IceCube 2): positions of IceCube holes drilled in the period of transient data taking, light gray circles (IceCube 1): other IceCube holes, black filled circles: locations of SPATS strings, triangles and square: location of RW. The elongated structures are discussed in Sec. 4.3 In (c) the depth distribution of acoustic events versus time since August 2008 is shown. The zero at the time axis corresponds to Jan 1, 2009 and drill periods are indicated in light gray. 


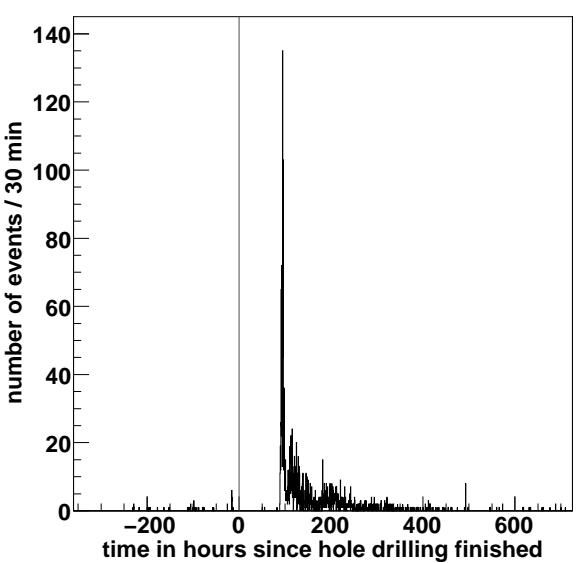

(a)

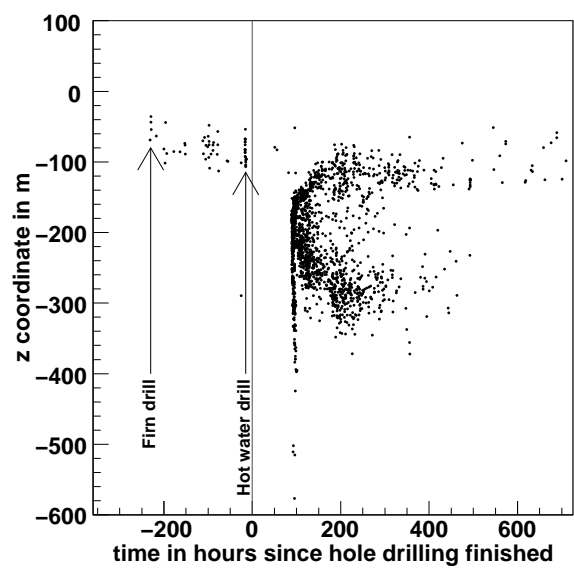

(b)

Figure 8: (a) Number of acoustic events per hour from hole 81 before and after drilling and (b) depth distribution versus time of acoustic events from hole 81 . The vertical line indicates the end of hot water drilling for hole 81 .

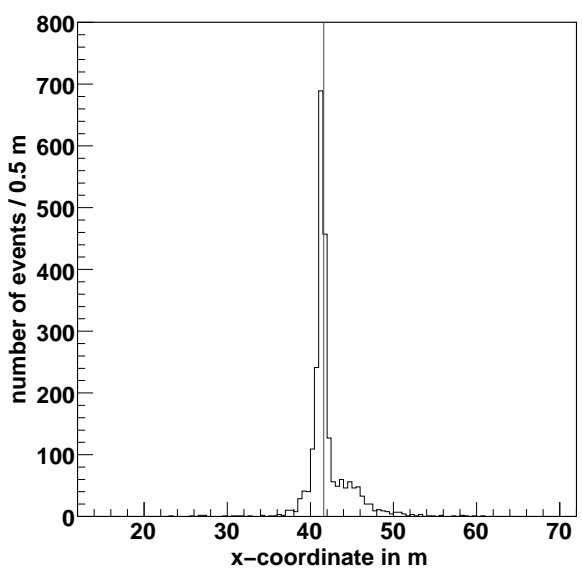

(a)

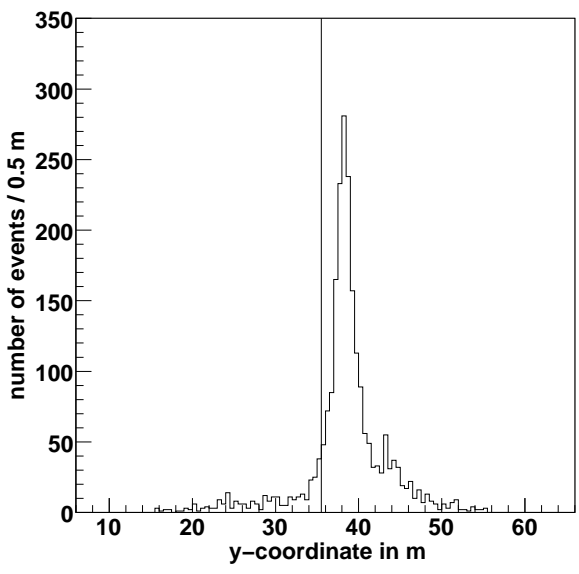

(b)

Figure 9: (a) $x$-coordinate and (b) $y$-coordinate distribution of acoustic events from hole 81 . The vertical line shows the nominal position of hole 81 


\subsubsection{Noise from Rodriguez-Wells}

When first acoustic events had been reconstructed during the first period from August to November 2008 (quiet period), a strong clustering in a certain region of the $x$-y plane at about $(-150 \mathrm{~m}, 300 \mathrm{~m})$ became visible. It was found that this was the position of the 2007/08 Rodriguez-Well used for the hot water drilling system.

This type of well has been introduced by Rodriguez and others in the early 1960s [10] to supply water from a glacier in Greenland. In Fig. 10, a sketch of the installation is shown. Hot water cycled by a pump system is used to melt ice below the firn layer at 60 to $80 \mathrm{~m}$ depth to maintain a fresh water reservoir. An expanding cavity is formed with a diameter as large as 15 to $20 \mathrm{~m}$. For IceCube and its predecessor AMANDA this technique is used in connection with drilling at the South Pole since mid 1990s. If the well is used a second time a year later, a second cavern is formed at a deeper level.

Having identified acoustic events arising from the 2007/08 Rodriguez-Well, three other event clusters were found, two of them could be attributed to other IceCube Rodriguez-Wells from 2006/07 and 2004 to 2006. The fourth event cluster turned out to be located at the probable position of the last AMANDA Rodriguez-Well used in the final two drilling seasons up to 2001. No documented coordinates could, however, be found for that position. Available information about acoustic event clusters connected with RodriguezWells is summarized in Table2. As can be seen from the table and from Fig. 11(a) the acoustic events from the two Rodriguez-Wells used only during one season are located at shallower depths than those from RodriguezWells used twice. This is in agreement with expectations from the sketch in Fig. 10 The former were seen to emit acoustic signals from regions of decreasing volume around the well core and finally stopped, the older one in October 2008, the younger one in May 2009. As an example, Fig. 12 shows the time profile of refreezing for Rodriguez-Well 2007/08, which was used only during one season. In contrast to that, acoustic events are observed until today from the six and ten years old deeper wells (see Fig. 11(b)). The mechanism of sound production in and around the Rodriguez-Well caverns is still under debate in particular for the older wells.

\subsection{Acoustic event simulation}

A simple acoustic transient event simulation is done by calculating the signal propagation times for the distance $d_{n}=\sqrt{\left(x_{n}-x\right)^{2}+\left(y_{n}-y\right)^{2}+\left(z_{n}-z\right)^{2}}$ between source (e.g. IceCube hole at $(x, y, z))$ and sensors $n=$ $1, \ldots, n_{\max }$ with $\Delta t_{n}=d_{n} / v_{s}$. The signal is transmitted

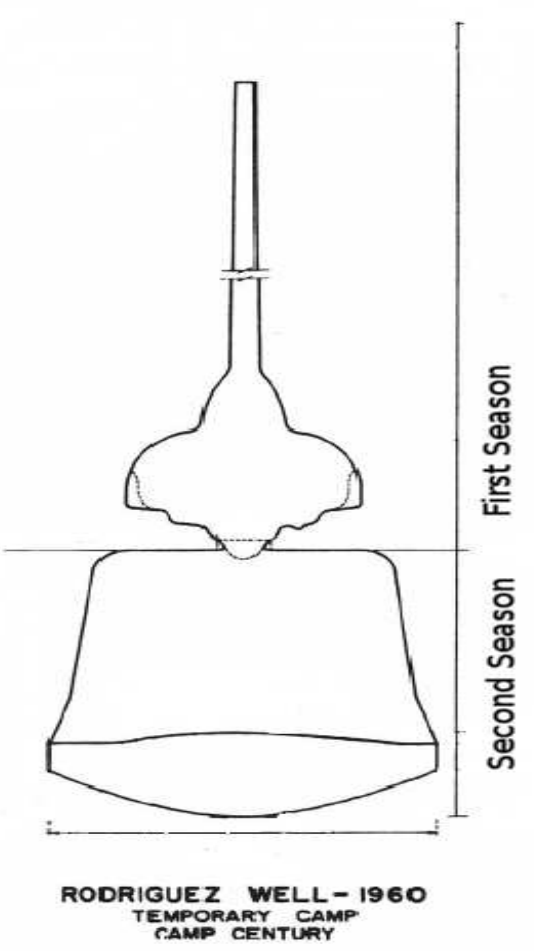

Figure 10: Section of the Camp Century well after a first and second season of operation (Schmidt and Rodriguez 1962) from [10].

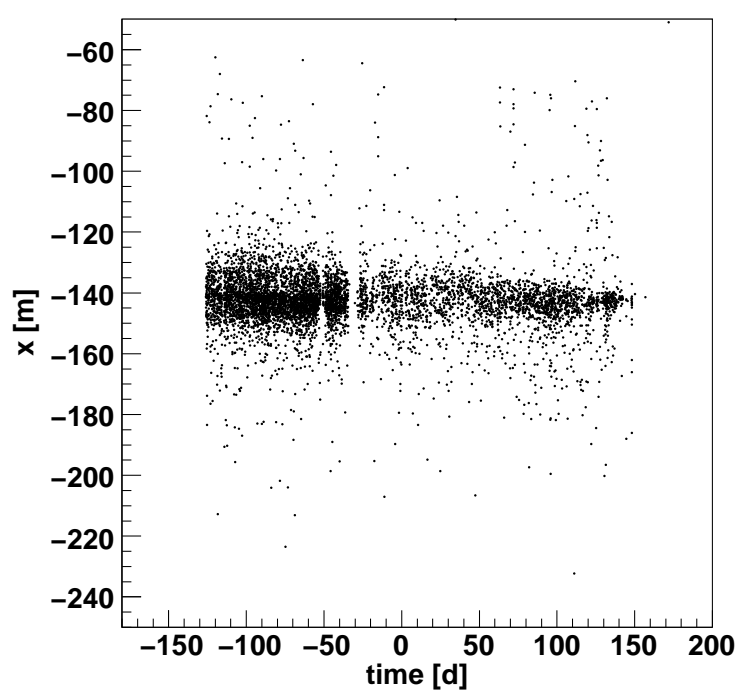

Figure 12: Re-freezing of Rodriguez-Well 2007/08 in the $x$ - $t$ plane. Acoustic signals are emitted from an decreasing area around the well core until they disappear in May 2009. The time is given in days relative to Jan. 1,2009 . 
Table 2: Positions of acoustic events from different Rodriguez-Wells. The errors are given by the rms values of the event distribution and the asterisk in the last column marks the date when our analysis stopped.

\begin{tabular}{|l|r|r|r|r|r|l|l|}
\hline Name & $x_{\text {nom }}[\mathrm{m}]$ & $y_{\text {nom }}[\mathrm{m}]$ & $x_{\text {fit }}[\mathrm{m}]$ & $y_{\text {fit }}[\mathrm{m}]$ & $z_{\text {fit }}[\mathrm{m}]$ & used & seen until \\
\hline \hline AMANDA & - & - & $276.2 \pm 0.4$ & $123.6 \pm 1.0$ & $-147.3 \pm 1.1$ & $2 \mathrm{y}$ & Feb. $10^{*}$ \\
\hline IC-RW 04-06 & 458.6 & 102.7 & $412.6 \pm 5.3$ & $124.0 \pm 2.4$ & $-147 \pm 11$ & $2 \mathrm{y}$ & Feb. $10^{*}$ \\
\hline IC-RW 06/07 & 297.2 & 254.4 & $279.6 \pm 0.4$ & $252.2 \pm 1.0$ & $-114.2 \pm 0.7$ & 1 y & Oct. 08 \\
\hline IC-RW 07/08 & -145.6 & 295.6 & $-138.6 \pm 0.4$ & $297.7 \pm 0.6$ & $-118.3 \pm 1.0$ & $1 \mathrm{y}$ & May 09 \\
\hline
\end{tabular}

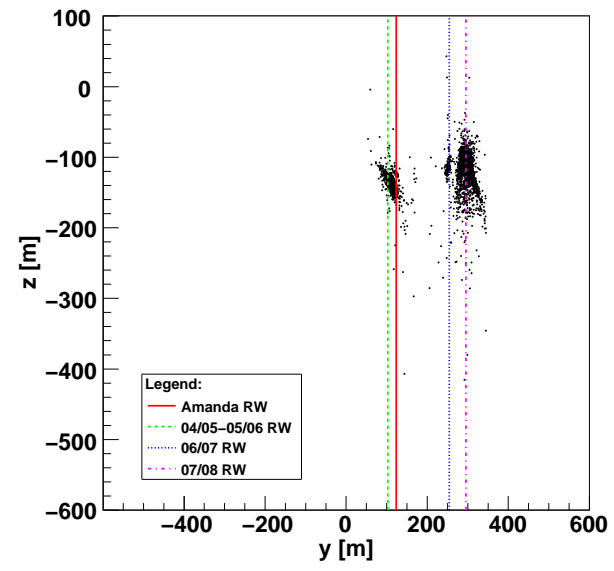

(a)

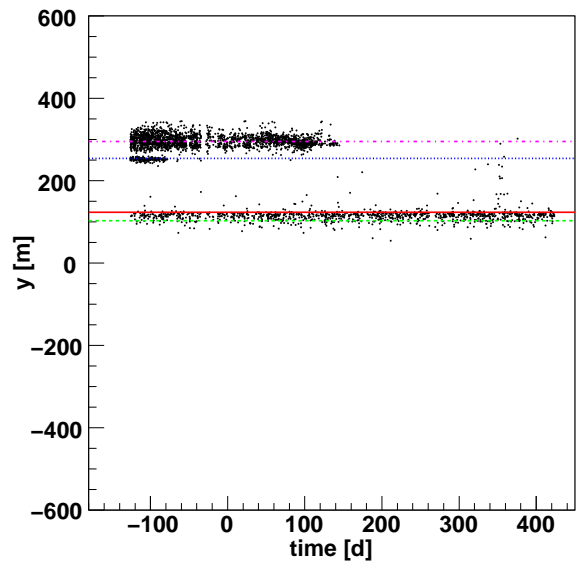

(b)

Figure 11: Acoustic events distribution in (a) depth versus $y$-coordinate and (b) in $y$-coordinate versus time. Lines: fitted positions of AMANDARW (solid red), 04/05-05/06 IceCube-RW (dashed green), 06/07 IceCube-RW (dotted blue), 07/08 IceCube-RW (dashed-dotted magenta). The time is given in days relative to Jan. 1, 2009. 


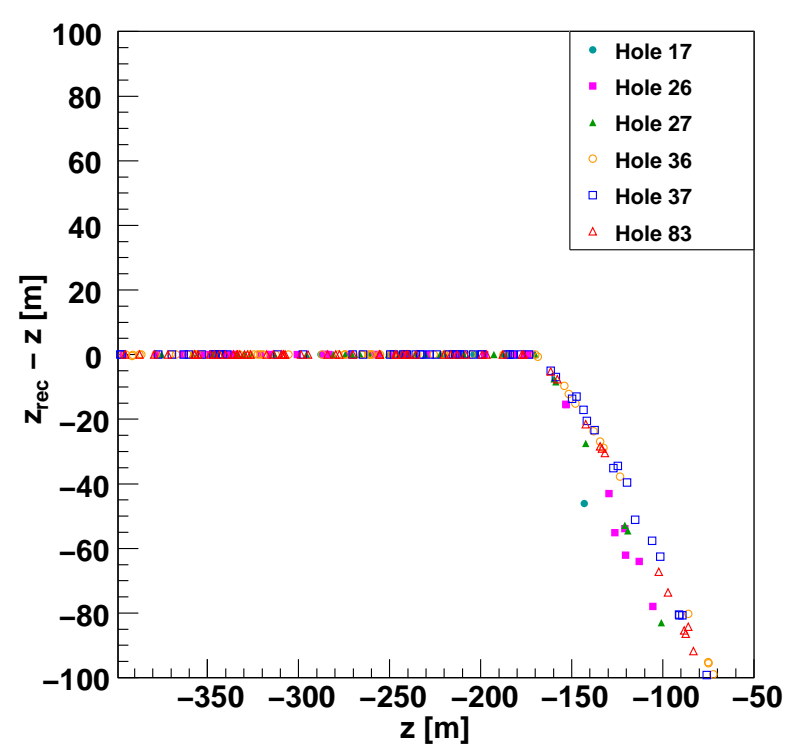

Figure 13: Difference between reconstructed event position and true event position for simulated events. Deviations seen in the upper region $(z>-170 \mathrm{~m})$ are caused by the depth dependent sound speed.

from a random point inside a certain cylindrical volume (radius $2 \mathrm{~m}$, depth $2000 \mathrm{~m}$ ) around the source.

Although knowing that the true IceCube hole diameter is about $0.7 \mathrm{~m}$, we take into account the possibility that tension cracks might appear outside the hole bounding surface, suggesting a larger simulation radius. The reconstruction of events simulated with constant speed of sound and without considering attenuation effects implies an exact source localization, which is in contradiction to the real data vertex results, where a specific spread of vertices around the source (Fig 7(b) and a lack of events below and above a certain depth is visible (Fig. 8(b)]. The major reason for misreconstruction of events at shallow depth $(-200 \mathrm{~m}<z<-1 \mathrm{~m})$ is the depth dependence of the sound speed [2], which is therefore included in the simulation. Above $174.8 \mathrm{~m}$ and below $1 \mathrm{~m}$ depth, the parameterization

$v_{s}=-\left(262.379+199.833\left|\frac{z}{\mathrm{~m}}\right|^{\frac{1}{2}}-1213.08\left|\frac{z}{\mathrm{~m}}\right|^{\frac{1}{3}}\right) \mathrm{m} \mathrm{s}^{-1}(3)$

is used. Below a constant sound speed value of $v_{s}=$ $3878 \mathrm{~m} \mathrm{~s}^{-1}$ is assumed. Parameterization of Eq. 3 is obtained using a fit of in-ice sound speed data points [9, 2]. These conditions are taken into account by integrating along the path between source and sensor using the parameterized value of $v_{s}$ at every point. Due to the absolute positions of the source and the sensors and their relative locations to each other, refraction effects are negligible as shown e.g. in Fig. 6 of reference [2] and are thus neglected in this paper. Further improvements are achieved by using additional information on the acoustic pressure wave attenuation in ice. We apply the formula

$$
S_{R}^{n}=\left(\frac{S_{0} \cdot d_{0}}{d_{n}}\right) e^{-\frac{d_{n}-d_{0}}{\lambda}},
$$

with the initial amplitude $S_{0}$ at the distance $d_{0}$ chosen to fit the real data and an attenuation length $\lambda=300 \mathrm{~m}$ as measured for South Pole ice with SPATS [3]. $S_{R}^{n}$ is the corresponding signal amplitude at the sensor $n$. If the signal strength at a sensor is above $\sim 300 \mathrm{mV}(5.2 \sigma$ above the noise level), a hit is triggered as in real data.

Good agreement between reconstructed real and reconstructed simulated events is obtained below $170 \mathrm{~m}$ depth, where the localisation precision in the $\mathrm{z}$ coordinate is $25 \mathrm{~cm}$ (see Fig 13). As expected, we observe a large influence of the depth dependence of the sound speed on reconstructions in the upper region of SPATS (between 0 and $170 \mathrm{~m}$ depth), as one can also see in Fig. 13. The significant deviation of the sound speed from the constant value used in the reconstruction explains the spread of vertices seen in the real data (see Fig. 7(b)], whereas the direction of this smearing is caused by the detector geometry. Due to the strong attenuation, it is more difficult to observe deep events, which is well reproduced by the simulation.

\section{Estimated neutrino flux limit}

Although SPATS has not been built to measure a relevant neutrino flux limit, it is interesting to find out how sensitive a corresponding measurement could be, using data from this setup. In order to determine the number of events not connected to IceCube construction activities in the sensitive region of SPATS, we omit the area of IceCube strings and the data from the drill periods. The area taken into account is indicated by the hatched area of Fig. 14(a). Furthermore, we look at depths between 200 and $1000 \mathrm{~m}$, in the region of constant speed of sound, to avoid the smearing effect in the reconstruction of acoustic event locations described in Section 4.3 . In the 245 days of transient data taking (quiet period 2) we found no events in the defined region. This observation is used to calculate an upper limit on the cosmogenic neutrino flux.

The effective target volume Fig. 14(b) was calculated following the approach used for the first acoustic neutrino limit estimate [11]. The neutrinos were assumed 
to be down-going and to be uniformly distributed on a $2 \pi$ half sphere. The total cross-section is taken from a function derived by extrapolation of measured cross sections to higher energies [20], which is valid for neutrino energies above $10^{5} \mathrm{GeV}$. Together with the interaction vertex, the direction $(\theta, \phi)$ defines the plane of the acoustic pressure wave perpendicular to this direction. The sensor observation angle was then calculated relative to this plane for each vertex. A number of $10^{7}$ events were simulated for neutrino energies $E_{v}$ from $10^{18} \mathrm{eV}$ to $10^{22} \mathrm{eV}$. The energy $E_{\text {had }}$ of the hadronic cascade was assumed to be a constant fraction $y=0.2$ of the neutrino energy, i.e. $E_{\text {had }}=0.2 E_{v}$. The contribution of the cascade originating from the final state electron in the electron-neutrino charged current reaction is omitted in the present model calculations, because its acoustic signal is expected to be small due to the LPMeffect [21]. The acoustic pressure $P_{\max }$ was calculated with respect to observation angle and distance. We use the Askaryan [12] model to calculate the acoustic signal strength assuming a cylindrical energy deposition in the medium of length $L$ and diameter $d$. No attempt was made to model angular sensitivity or frequency response of the sensor. A minimum threshold of $\sim 300 \mathrm{mV}$, as in the real SPATS measurement, was applied. Using our estimate for the average SPATS sensor sensitivity (Sec.3.3), this transforms to a necessary minimum pressure of $\sim 70 \mathrm{mPa}$. At least five hits distributed over all four strings were required for an event to trigger.

The procedure has a number of rather large associated uncertainties that are discussed in the following list:

- The biggest uncertainty is related to the different predictions of the various thermo-acoustic models for the acoustic signal strength (see e.g. [17], [18]). We assume a $100 \%$ uncertainty on this quantity. No absolutely calibrated measurement exists so far in any medium to fix that problem.

- The Landau-Pomeranchuk-Migdal effect [19] adds an additional uncertainty by changing the cross sections of bremsstrahlung and pair-production at ultra-high energies. The effect elongates preferentially primary electron cascades from neutrino interactions and diminishes expected acoustic signals [21]. It starts to become important for hadronic cascades above $10^{18} \mathrm{eV}$. Above $10^{20} \mathrm{eV}$ its influence is diminished by photo-nuclear and electro-nuclear interactions (see [22] for a recent detailed discussion).

- The uncertainty of about $40 \%$ in the absolute noise level determination leads to a corresponding uncer- tain trigger threshold. This makes the lower energy threshold for contributing neutrino interactions uncertain.

- The angular efficiency loss of the single sensor channels is elaborated by use of a data set where two channels per sensor are available. With the threshold taken $(70 \mathrm{mPa})$, an efficiency of $>99 \%$ is found for $99 \%$ of the azimuthal angular range. This leads to the conclusion that the azimuthal efficiency loss is negligible in comparison to other error sources taken into account.

- The $30 \%$ error on the sound attenuation length is of minor importance in comparison to the effects discussed above.

These problems influence all acoustic (and partly radio) neutrino limits given so far (see Fig. 14(c)). In the present paper the acoustic signal is calculated with a model delivering signal values in the middle between extreme predictions.

The observation of zero events inside the effective volume of SPATS gives an upper limit of $N_{\text {obs }}=2.44$ events at a Poissonian $90 \%$ confidence level [23]. The flux limit for an assumed trigger threshold of the measurement of $70 \mathrm{mPa}$ is shown in Fig. 14(c) as dashed curve together with cosmogenic neutrino flux predictions and results from other experiments. The grey and dark grey bands around the given limit indicate the uncertainties of this quantity due to the uncertainties discussed above. The upper border of the grey shaded area can therefore be considered as a conservative neutrino flux limit derived from the SPATS data, provided that the assumptions made in this work for estimating the detector sensitivity hold.

\section{Summary and Outlook}

We presented an analysis of acoustic noise data recorded with the South Pole Acoustic Test Setup (SPATS) in the deep Antarctic ice at the geographic South Pole. We found the absolute noise level to be extremely stable over time. Its estimated magnitude of $14 \mathrm{mPa}$ in the deep ice, below $200 \mathrm{~m}$, is comparable to the noise in the deep sea when weather conditions are calm [4, 5, 6]. Studies of transient noise in the SPATS data revealed the refreezing IceCube holes and Rodriguez-Wells as sources. The high quality of the data allowed us to study the refreezing processes as function of time in great detail. No transient acoustic signals in the deep ice were observed outside the instrumented volume of IceCube at depths below $200 \mathrm{~m}$. This 


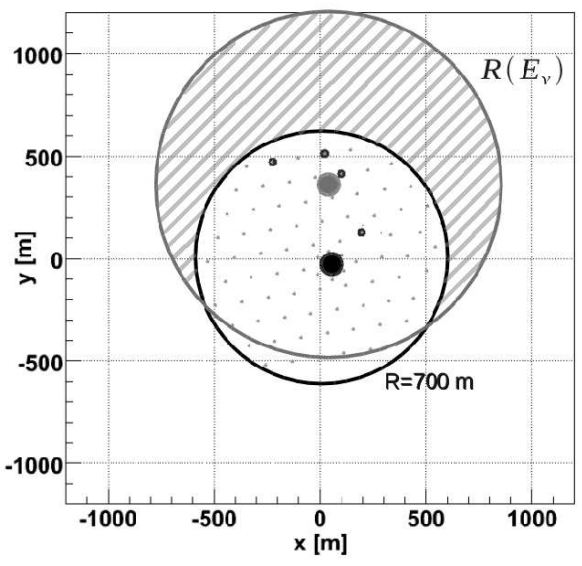

(a)

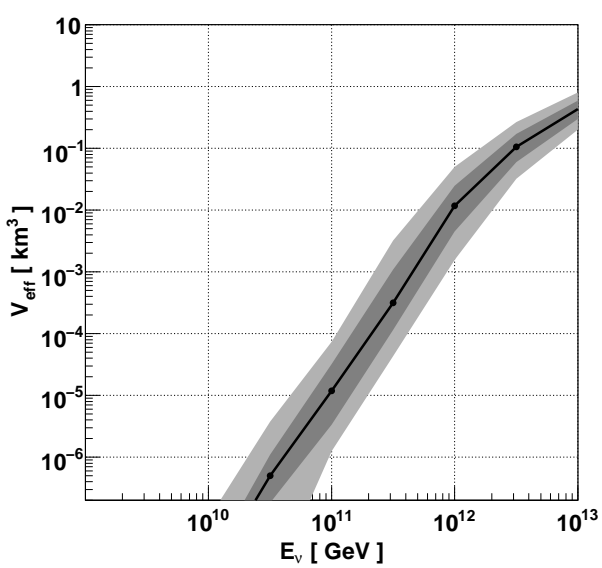

(b)

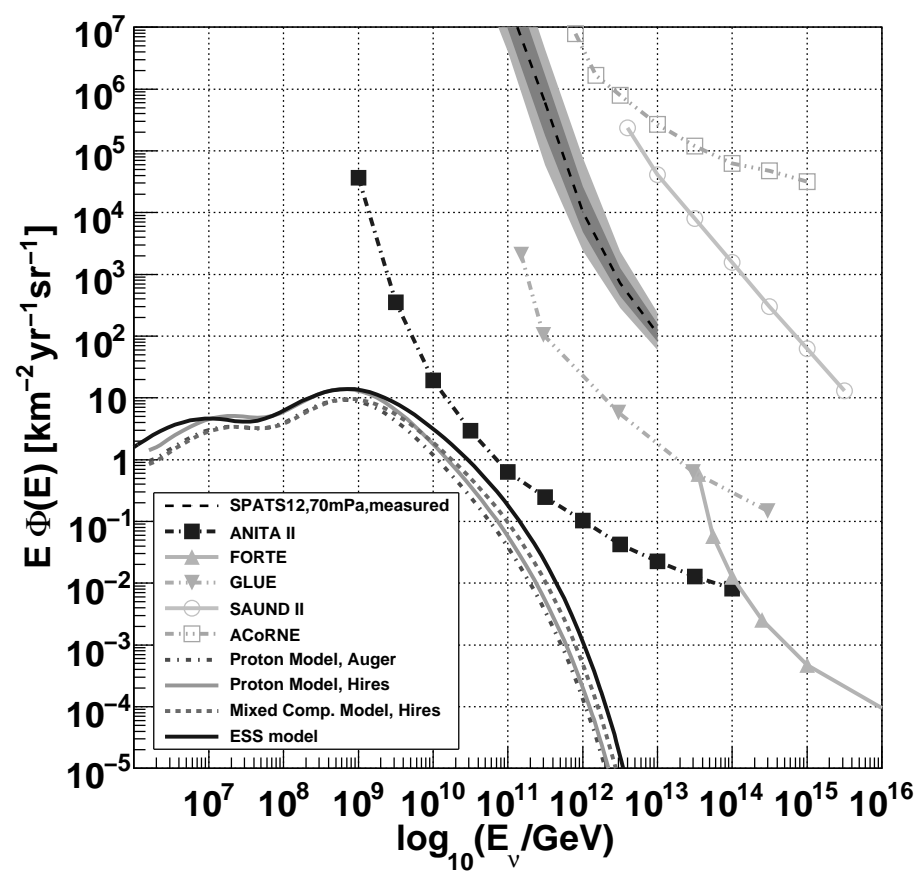

(c)

Figure 14: (a) Shown are the IceCube center (large black dot) with the construction area (black circle) and the SPATS center (large grey dot) with the sensitive area for acoustic detection (grey circle). In addition, strings with acoustic sensors (medium size dots) and all IceCube strings (small dots) are drawn in. To avoid events from IceCube construction, only the shaded area of the SPATS sensitive region is used in order to measure the neutrino flux limit. (b) Corresponding effective volume and (c) the neutrino flux limit of the 2009 SPATS configuration (70 mPa threshold, $\geq 5$ hits per event). The dark gray band (50 to $100 \mathrm{mPa}$ threshold) around the effective volume and around the limit considers uncertainties in absolute noise. The even broader light gray band includes additional uncertainties due to the choice of different acoustic models. Experimental limits on the flux of ultra high energy neutrinos are from ANITA II [13], FORTE [14], GLUE [15], SAUND II [26], ACoRNE [16]. For model reference see [24, 25]. 
enabled us to derive a first upper limit on the flux of ultra-high energy neutrinos with an acoustic detector in glacial ice.

SPATS is continuing to take data. An upgrade of the DAQ software to read out all sensor channels simultaneously and to form a multiplicity trigger online, will increase the detector sensitivity.

\section{Acknowledgements}

We acknowledge the support from the following agencies: U.S. National Science Foundation-Office of Polar Programs, U.S. National Science FoundationPhysics Division, University of Wisconsin Alumni Research Foundation, the Grid Laboratory Of Wisconsin (GLOW) grid infrastructure at the University of Wisconsin - Madison, the Open Science Grid (OSG) grid infrastructure; U.S. Department of Energy, and National Energy Research Scientific Computing Center, the Louisiana Optical Network Initiative (LONI) grid computing resources; National Science and Engineering Research Council of Canada; Swedish Research Council, Swedish Polar Research Secretariat, Swedish National Infrastructure for Computing (SNIC), and Knut and Alice Wallenberg Foundation, Sweden; German Ministry for Education and Research (BMBF), Deutsche Forschungsgemeinschaft (DFG), Research Department of Plasmas with Complex Interactions (Bochum), Germany; Fund for Scientific Research (FNRS-FWO), FWO Odysseus programme, Flanders Institute to encourage scientific and technological research in industry (IWT), Belgian Federal Science Policy Office (Belspo); University of Oxford, United Kingdom; Marsden Fund, New Zealand; Japan Society for Promotion of Science (JSPS); the Swiss National Science Foundation (SNSF), Switzerland; A. Groß acknowledges support by the EU Marie Curie OIF Program; J. P. Rodrigues acknowledges support by the Capes Foundation, Ministry of Education of Brazil.

\section{References}

\section{References}

[1] Y. Abdou et al., Design and performance of the South Pole Acoustic Test Setup, submitted to Nucl. Instrum. Meth. A, arXiv:1105.4339 [astro-ph.IM]

[2] R. Abbasi et al., Measurement of sound speed vs. depth in South Pole ice for neutrino astronomy, Astropart. Phys. 33 (2010) 277.

[3] R. Abbasi et al., Measurement of Acoustic Attenuation in South Pole ice, Astropart. Phys. 34 (2011) 382.

[4] J. A. Aguilar et al., AMADEUS - The acoustic neutrino detection test system of the ANTARES deep-sea neutrino telescope, Nucl. Instrum. Meth. A626-627 (2011) 128.
[5] G. Riccobene et al., Long-term measurements of acoustic background noise in very deep sea, Nucl. Instrum. Meth. A604 (2009) 149.

[6] V. Aynutdinov et al., Acoustic search for high-energy neutrinos in the Lake Baikal: Results and plans, Nucl. Instrum. Meth. A, in press, doi:10.1016/j.nima.2010.11.153.

[7] A. Achterberg et al., First year performance of the IceCube neutrino telescope, Astropart. Phys. 26 (2006) 155.

[8] R. J. Urick, Principles of underwater sound, 3rd edition, Peninsula Publishing (1983).

[9] J. G. Weihaupt, Seismic and gravity studies at the South Pole, Geophysics 284 (1963) 582.

[10] R. P. Schmitt and R. Rodriguez, Glacier water supply and sewage disposal systems, Proceedings of the Symposium on Antarctic Logistics (1962) 329, Boulder, Colorado, National Academy of Sciences, National Research Council.

[11] J. Vandenbroucke, G. Gratta and N. Lehtinen, Experimental study of acoustic ultra-high-energy neutrino detection, Astrophys. J. 621 (2005) 301.

[12] G. A. Askaryan, B. A. Dolgoshein, A. N. Kalinovsky and N. V. Mokhov, Acoustic detection of high energy particle showers in water, Nucl. Inst. Methods 164 (1979) 267.

[13] P. W. Gorham et al., Observational constraints on the ultrahigh energy cosmic neutrino flux from the second flight of the ANITA experiment, Phys. Rev. D82 (2010) 022004. Erratum: arXiv: 1011.5004 [astro-ph.HE].

[14] N. G. Lehtinen, P. W. Gorham, A. R. Jacobson and R. A. Roussel-Dupre, FORTE satellite constraints on ultra-high energy cosmic particle fluxes, Phys. Rev. D69 (2004) 013008.

[15] P. W. Gorham et al., Experimental limit on the cosmic diffuse ultrahigh-energy neutrino flux, Phys. Rev. Lett. 93 (2004) 041101.

[16] S. Bevan, Data analysis techniques for UHE acoustic astronomy, Nucl. Instrum. Meth. A604 (2009) 143.

[17] A. V. Butkevich et al., Prospects for radio-wave and acoustic detection of ultra- and superhigh-energy cosmic neutrinos (cross sections, signals, thresholds), Phys. Part. Nucl. 29 (1998) 266.

[18] S. Bevan et al., Study of the acoustic signature of UHE neutrino interactions in water and ice, Nucl. Instrum. Meth. A607 (2009) 398.

[19] A. B. Migdal, Bremsstrahlung and pair production in condensed media at high energies, Phys. Rev. 103 (1956) 1811

[20] J. P. Ralston, D. W. McKay, and G. M. Frichter, The ultra high energy neutrino nucleon cross section, arXiv:astro-ph/9606007

[21] V. Niess and V. Bertin, Underwater acoustic detection of ultra high energy neutrinos, Astropart. Phys. 26 (2006) 243.

[22] L. Gerhardt and S. R. Klein, Electron and photon interactions in the regime of strong LPM suppression, Phys. Rev. D82 (2010) 074017.

[23] G. J. Feldman and R. D. Cousins, A unified approach to the classical statistical analysis of small signals, Phys. Rev. D57 (1998) 3873.

[24] R. Engel, D. Seckel and T. Stanev, Neutrinos from propagation of ultra-high energy protons, Phys. Rev. D64 (2001) 093010.

[25] ftp://ftp.bartol.udel.edu/seckel/ess-gzk/2008

[26] N. Kurahashi, J. Vandenbroucke and G. Gratta, Search for acoustic signals from ultra-high energy neutrinos in $1500 \mathrm{~km}^{3}$ of sea water, Phys. Rev. D82 (2010) 073006. 